\title{
Du nouveau sur le domaine catalytique des protéine kinases
}

Les protéine kinases, qu'elles phosphorylent leurs cibles protéiques sur des sérine/thréonines ou sur des tyrosines, possèdent en commun de courtes séquences consensus, espacées de façon similaire, qui définissent un "cœur catalytique " d'environ 260 résidus [1]. La détermination de la structure tridimensionnelle de la sous-unité catalytique (C) de la protéine kinase dépendante de l'AMPc (PKA) a montré que ce domaine catalytique est composé d'un petit lobe portant le site de fixation de l'ATP, et d'un grand lobe qui contient les résidus impliqués dans la fixation du substrat protéique et la plupart de ceux impliqués dans le transfert catalytique du $\gamma$-phosphate [2]. La position relative des deux lobes n'est pas fixe: l'enzyme existe sous une configuration "ouverte" en absence de substrats ou "fermée" en présence d'ATP qui se trouve enfoui dans le complexe ternaire $[3,4]$. Dans de très nombreux cas, l'activité du domaine catalytique des protéines kinases est aussi réglée par d'autres protéines, comme par exemple la sous-unité régulatrice (R) pour la PKA ou les cyclines pour les protéine kinases liées au cycle cellulaire de la famille des cdc ou des cdk. Ces protéines, non catalytiques, agissent en se fixant sur la protéine kinase cible, mais on ne connaît pas le détail de ces interactions.

Les protéine kinases comportent en général des séquences extérieures au domaine catalytique qui, par définition, ne présentent pas de fortes homologies entre elles. Dans la PKA de mammifères, la séquence aminoterminale qui précède immédiatement le début du domaine catalytique correspond à une hélice $\alpha$ (l'hélice $\alpha A$ ) qui s'ajuste avec pré- cision à la face du domaine catalytique opposée à celle sur laquelle se fixent les peptides substrats. Chez Dictyostelium, un organisme très ancien du point de vue phylogénétique, la séquence de la sous-unité C de la PKA est très similaire à celle de la souris dans le domaine catalytique, mais elle comporte une longue extension amino-terminale. Véron et al. [5] ont observé la présence, près du $\mathrm{N}$-terminal de cette protéine, d'une séquence pouvant constituer une hélice équivalente à l'hélice $\alpha A$ de la sous-unité $C$ de souris. La modélisation moléculaire de la PKA de Dictyostelium sur les coordonnées atomiques de la PKA de souris indique que, dans les deux protéines, un motif de type " hélice $\alpha A$ ", extérieur au domaine catalytique dans la séquence, vient s'ajuster étroitement à celui-ci: la chaîne latérale d'un résidu tryptophane de l'hélice $\alpha \mathrm{A}$ pénètre profondément dans une poche hydrophobe du domaine catalytique, précisément là où s'articulent les deux lobes.

L'association de l'hélice $\alpha \mathrm{A}$ avec le domaine catalytique, conservée dans deux PKA très distantes du point de vue évolutif, se retrouve-t-elle dans d'autres membres de la superfamille des protéines kinases? Les auteurs ont trouvé un motif correspondant à une "hélice $\alpha A$ " dans la plupart de ces enzymes, notamment dans Src et les protéines kinases qui lui sont homologues. La modélisation de l'hélice $\alpha A$ de Src qui connecte le domaine $\mathrm{SH} 2$ avec le domaine catalytique dans la séquence primaire, permet de faire une prédiction forte sur les positions relatives de ces deux domaines: le domaine SH2 se place à proximité de l'extrémité C-terminale et de la tyro- sine 517 , ce qui établit une base structurale pour l'inhibition de l'activité de Src par phosphorylation de ce résidu crucial.

La conservation du motif de l'hélice $\alpha A$ dans la plupart des protéines kinases pose la question de son rôle fonctionnel. Les données expérimentales précises pour résoudre cette question manquent encore, mais il est remarquable que le domaine du cœur catalytique sur lequel s'ajuste l'hélice $\alpha \mathrm{A}$ est constitué d'une grande surface très plane et particulièrement hydrophobe qui pourrait constituer un site de fixation de protéines effectrices.

M.V.

1. Hanks SK, Quinn AM, Hunter T. The protein kinase family: conserved features and deduced phylogeny of the catalytic domain. Sirience 1988; 241: 42-52.

2. Knighton DR, Zhen J, Eyck I.FT, Ashford VA, Xuong N, Taylor SS, Sowadski JM. Crystal structure of the catalytic subunit of cAMPdependent protein kinase. Science 1991; 253 : 407-20.

3. Bossemeyer D, Engh RA, Kinzel V, Pontingl H, Huber R. Phosphotransferase and substrate binding mechanism of the cAMP dependent protein kinase catalytic subunit from porcine heart as deduced from the 2.0 A structure of the complex with $\mathrm{Mn}^{+}$ adenylyl imidodiphosphate and inhibitor peptide PKI(5-24). EMBO J 1993; 12: 849-59. 4. Zheng J, Knignton DR, Ten Eyck I.F, Karlsson R, Xuong N, Taylor SS, Sowadski JM. Crystal structure of the catalytic subunit of cAMP-dependent protein kinase complexed with MgATP and peptide inhibitor. Biochemistr 1993; 32: 2154-61.

5. Véron M, Radsio-Andzelm E, Tsigelny 1 , Ten Eyck I.F, Taylor SS. A conserved helix motif complements the protein kinase core Proc Nall Acad Sci USA 1993; 90): 10618-22. 